Research Article

\title{
Parallax Barrier for Weakening Vernier Fringe in Naked-Eye LED 3D Display
}

\author{
Hong-Ran Zeng and Wu-Xiang Zhao \\ School of Electronic and Information Engineering, Sichuan University, Chengdu 610065, China \\ Correspondence should be addressed to Wu-Xiang Zhao; zhaowuxiang@scu.edu.cn
}

Received 31 July 2021; Accepted 4 December 2021; Published 22 December 2021

Academic Editor: E. Bernabeu

Copyright (c) 2021 Hong-Ran Zeng and Wu-Xiang Zhao. This is an open access article distributed under the Creative Commons Attribution License, which permits unrestricted use, distribution, and reproduction in any medium, provided the original work is properly cited.

In naked-eye LED 3D display, vernier fringe is apparent for a conventional parallax barrier. This paper presents an intended misplaced parallax barrier with discrepant width of Light Translucent Slits (LTSs) to weaken vernier fringe. Because of the wild Black Matrix (BM) of LED display, which causes apparent vernier fringe, we enlarge the width of the LTS and move the slits properly in their periods. This structure increases the periodic difference between the parallax barrier and pixel of the LED display, which can increase the brightness of the diazone of vernier fringe and make it to appear more sparsely. In this way, vernier fringe produced by those two periods is weakened at the condition that no obvious crosstalk of stereoimages is increased. The performances of simulation and experimental display prototype show that the diazone of vernier fringe is faded and obviously sparser in the naked-eye LED 3D display. As a result, vernier fringe of this display is significantly decreased and not visible for viewing.

\section{Introduction}

Naked-eye 3D display technologies do not need any accessory such as stereo glasses and is currently a research hotspot in the field of display $[1,2]$. Among them, the LCDbased parallax barrier naked-eye 3D display is a popular experimental scheme with simple structure, easy realization, and low cost, which has become a relatively mature product in recent years [3-7]. However, the LCD display screen is hard to be enlarged due to its low brightness, while the LED screen is suitable for large-screen display due to its high brightness, large size, and bright color [8-12]. For the traditional vertical parallax barrier design, the LTS is placed parallel to the column pixels of the display screen $[13,14]$. When the eye sight captures the BM, which is the nonluminous part between adjacent subpixels, through the LTS, it will generally trigger the obvious periodic black and white gradient slits, named as vernier fringe [15-17], bringing discomfort to the viewers and seriously affecting the display effect. The use of an oblique parallax barrier can impair vernier fringe, but it will significantly increase the crosstalk of the stereoimage [18-21]. In order to reduce the discomfort brought by vernier fringe and not significantly increase crosstalk, this paper proposes a designing method of misplaced parallax barrier with nonuniform width of the LTS based on the wide characteristics of BM of the LED screen [22]. By appropriately increasing the width of the LTS of the parallax barrier and moving it during the period, the difference between the periodic structure of the parallax barrier and the pixel periodic structure of the LED screen can be increased, so as to reduce the correlation between the spectral element and the display screen [23] and further enhance the brightness of diazone in vernier fringe and make it sparse. Therefore, the proposed method can attenuate the vernier fringe without obvious introduction of crosstalk of the stereoimage.

\section{The Structure and Principles of the Parallax Barrier Naked-Eye 3D Display}

We take the front-parallax barrier naked-eye 3D displayer based on the LED screen as an example to illustrate the inner structure and principle [24], which are shown in Figure 1. We set the spacing among adjacent pixels in the LED screen 


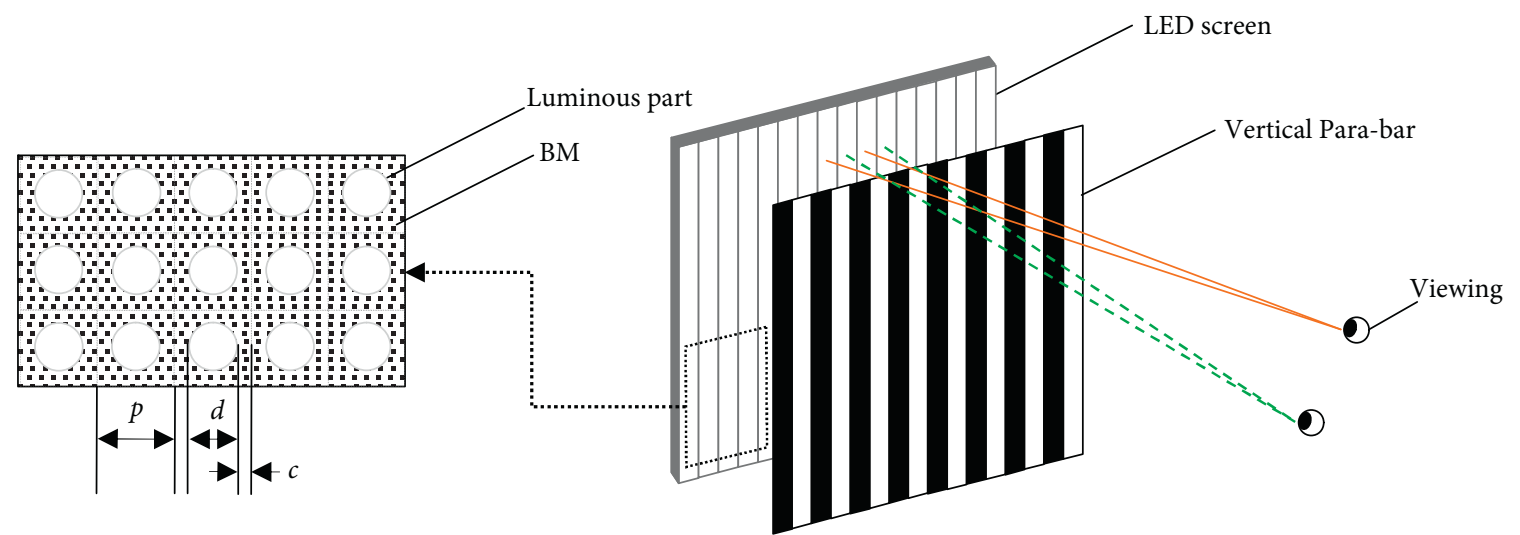

(a)

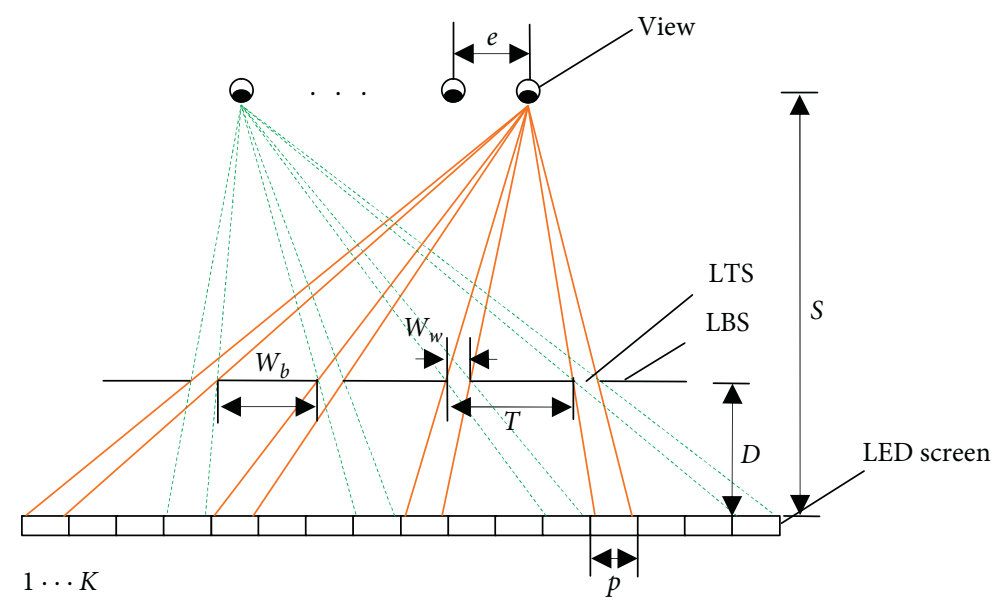

(b)

FIGURE 1: Naked-eye 3D display based on the parallax barrier. (a) Structure; (b) principle of beam split.

as $p$, the width of the LTS of the parallax barrier (parabar) as $W_{w}$, Light Blocking Slit (LBS) width as $W_{b}$, period as $T=W_{w}+W_{b}$, optimal viewing distance as $S$, and the distance between adjacent viewpoints as $e$, according to the average human pupil distance. In terms of the triangle similarity principle, the relationship between the abovementioned parameters can be given by

$$
\begin{aligned}
D & =\frac{p \cdot S}{e+p}, \\
W_{w} & =\frac{p \cdot e}{e+p}, \\
T & =\frac{K \cdot e \cdot p}{e+p} .
\end{aligned}
$$

\section{Design of a Misplaced Parallax Barrier with Nonuniform LTS Width}

In the parallax barrier naked-eye 3D display, since the pixels of the 2D displaying board are arranged in an orderly periodic matrix structure, the light of pixels interferes with the periodic parallax barrier in front of the pixel panel to form obvious vernier fringe, which makes the $3 \mathrm{D}$ display effect terrible to watch. Thus, in order to weaken the vernier fringe from the traditional vertical parallax barrier, this paper proposes a designing method of the parallax barrier with nonuniform width of LTS. As shown in Figure 2, the pixel spacing of the LED screen is denoted as $p=2.5 \mathrm{~mm}$ and the white disc, whose diameter equals to $1.5 \mathrm{~mm}$, represents the actual luminous part of the pixel. In addition, half width of the $\mathrm{BM}$ is set as $0.5 \mathrm{~mm}$, noted by $c=0.5 \mathrm{~mm}$, and the number of viewing point is set to $K=5$. Also, the viewing distance $\mathrm{S}$ is defined as $4 \mathrm{~m}$, and the adjacent distance of viewing points $e$ is $65 \mathrm{~mm}$.

First and foremost, three important parameters of the traditional vertical parallax barrier, LTS width, period, and distance from the parallax barrier to the LED screen, can be obtained by substituting the abovementioned parameters into equations (1)-(3): $W_{w}=2.41 \mathrm{~mm}, T=12.04 \mathrm{~mm}$, and $D=148.15 \mathrm{~mm}$. Then, keeping the period $T=12.04 \mathrm{~mm}$ unchanged, we alter the width of the LTS and move to the corresponding position in its respective period. However, there must be an upper limit $W_{w \max }$ and lower limit $W_{w \text { min }}$ to the LTS width to ensure the effect of stereodisplay not to increase too sharply due to the mighty wide LTS and not to cause serious loss of stereoimage brightness due to excessive narrow LTS. The experiment verifies that the vernier fringe can be effectively weakened and the 


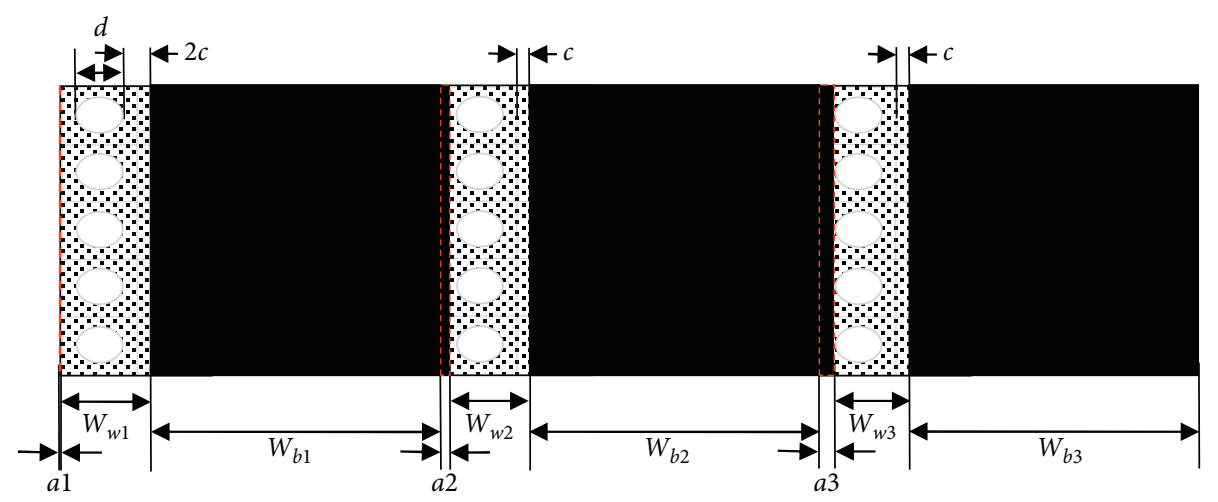

FIGURE 2: Structure of the malposed parallax barrier with discrepant width of slits, $W_{w 1}=3.0 \mathrm{~mm}, W_{w 2}=2.7 \mathrm{~mm}$, and $W_{w 3}=2.41 \mathrm{~mm}$, respectively.

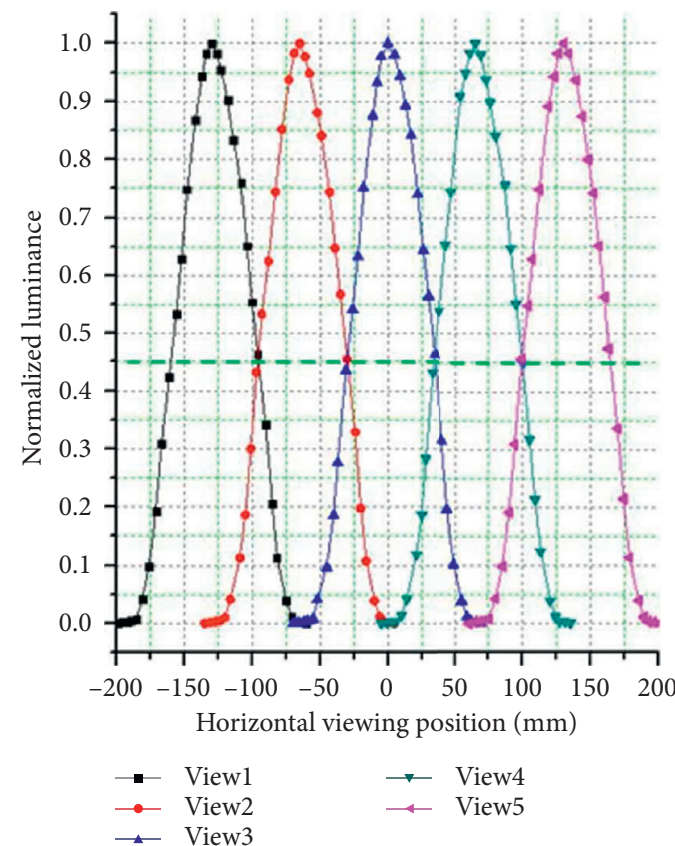

(a)

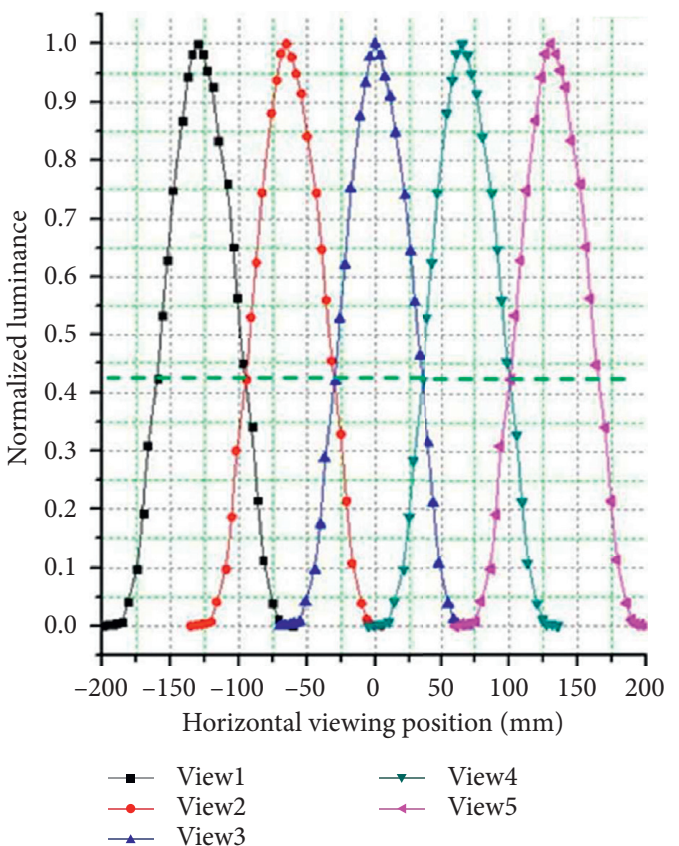

(b)

FIgURE 3: Normalized brightness distribution curves of naked-eye 3D displays based on the parallax barrier at the optimum 3D view distance. (a) Using the proposed parallax barrier; (b) using the conventional vertical parallax barrier.

crosstalk has little effect when the width of the LTS is equal to the sum of the pixel spacing $p$ and the half width $c$ of the $\mathrm{BM}$. The crosstalk can increase dramatically if the width of the LTS is further expanded. Besides, the width of the LTS equaling to the original width, $W_{w}=2.41 \mathrm{~mm}$, is the minimal width which we can implement as the brightness of the stereoimage can be radically disrupted by the deteriorative vernier fringe when smaller width is applied. Thus, the upper limit of LTS width is $W_{w \max }=p+c=3 \mathrm{~mm}$, and the lower limit is $W_{w \min }=W_{w}=2.41 \mathrm{~mm}$.

During the experiment, the widths of the LTS in the first period $T_{1}$ and the second period $T_{2}$ are extended to $W_{w 1}=p+c=3 c+d=3.0 \mathrm{~mm}$ and $W_{w 2}=2.7 \mathrm{~mm}$, shifting in the right direction $a_{1}=0 \mathrm{~mm}$ and $a_{2}=0.3 \mathrm{~mm}$, respectively, whereas the width of the LTS in the third period T3 remains the same, $W_{w 3}=W_{w}=2.41 \mathrm{~mm}$, shifting to the right $a_{3}=0.59 \mathrm{~mm}$. Finally, the experimental process is replicated over every three periods to complete the proposed designing means of the misplaced parallax barrier with nonuniform width of LTS.

\section{Simulation and Experimental Results}

4.1. Simulation Results and the Analysis. We use ASAP software to simulate the brightness distribution of every view point for the naked-eye 3D display. Figure 3 illustrates the normalized luminance distribution in the horizontal direction at the optimum viewing distance. Figure 3(a) shows the simulation performance of the proposed misplaced parallax barrier with nonuniform LTS width, and Figure 3(b) demonstrates the corresponding performance of the conventional vertical parallax barrier. 
TABLE 1: Specifications and parameters of the two prototypes, prototype 1 with a misplaced parallax barrier with nonuniform width of the LTS and prototype 2 with a traditional vertical parallax barrier.

\begin{tabular}{lcc}
\hline Parameters & Prototype1 & Prototype2 (mm) \\
\hline Size & & $640 \times 320 \mathrm{~mm}$ \\
2D resolution & $256 \times 128$ \\
Pixel spacing $p$ & $2.5 \mathrm{~mm}$ \\
Number of viewing points $K$ & 5 & $4 \mathrm{~m}$ \\
Optimal viewing distance $S$ & $51 \times 128$ \\
3D resolution & $3.0 \mathrm{~mm}$ & $12.04 \mathrm{~mm}$ \\
Period & $2.7 \mathrm{~mm}$ & 2.41 \\
& $2.41 \mathrm{~mm}$ & 2.41 \\
LTS's width & & 2.41 \\
\hline
\end{tabular}

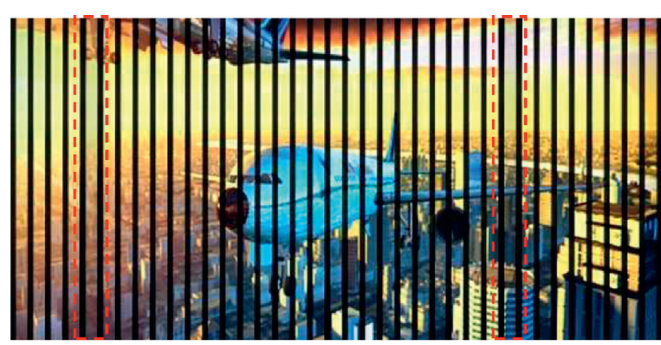

(a)

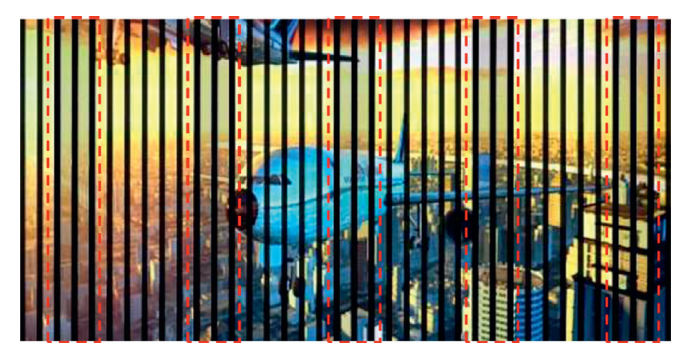

(b)

FIgURE 4: Comparison of experimental results. (a) Diazone of prototype 1: light, thin, and sparse; (b) diazone of prototype 2: prominent, wide, and dense.

More specifically, the horizontal axis of Figure 3 represents the horizontal viewing position and the viewing position is directly opposite the center of the naked-eye 3D display when the value on horizontal axis reaches zero $(0 \mathrm{~mm})$. We can qualitatively measure the severity of crosstalk by the amount of the overlapped region of the normalized luminance distribution at each view point. The smaller the overlapped area is, the less the crosstalk is introduced and, thereby, the better the performance of the naked-eye 3D displayer is. Comparing Figure 3(a) with Figure 3(b), the overlapped region of the brightness curves of each view point in Figure 3(a) is slightly more than that in Figure 3(b), enhancing the crosstalk merely a little bit. But, it influences little on the visual zone and viewing effect.

4.2. Experimental Results and Analysis. To conduct the comparative experiments, a naked-eye $3 \mathrm{D}$ displaying prototype 1 with a misplaced parallax barrier with nonuniform width of the LTS and a naked-eye 3D display prototype 2 with a traditional vertical parallax barrier were produced. The dominant parameters are shown in Table 1. Also, Figure 4 demonstrates the image details of vernier fringe when two prototypes display the stereoimage, while Figure 4(a) implies applying the prototype 1 with a misplaced parallax barrier with nonuniform width of the LTS, whereas we adopt the traditional prototype 2 for Figure 4(b). The consequences show that the diazone of vernier fringe is light, thin, and sparse, giving rise to the desired viewing effect for prototype 1, but in Figure 4(b), it is prominent, wide, and dense.

Therefore, the experiment results verify that the proposed structure can obviously reduce the vernier fringe of the parallax barrier naked-eye 3D display and improve the stereoimaging effect. By appropriately increasing the width of the LTS of the parallax barrier and moving it to the corresponding position in its period, the difference between the periodic structure of the parallax barrier and the pixel periodic structure of the LED screen can be enlarged. Hence, the correlation between the splitter elements and the displaying screen can be lessened. Such a design method reduces the contrast ratio of the $\mathrm{BM}$ at the junction of adjacent viewpoints, thus promoting the brightness of the diazone in vernier fringe and making it sparse.

\section{Conclusions}

In this paper, in order to effectively reduce the vernier fringe of the parallax barrier naked-eye 3D display, a new designed parallax barrier is proposed, which is the misplaced parallax barrier with nonuniform width of the LTS. This parallax barrier can enhance the brightness of the diazone in vernier fringe and make it obviously fade, thin, and sparse. The simulation and experimental results prove that the proposed structure can effectively weaken vernier fringe at the condition that no obvious crosstalk of stereoimages is increased. Thus, the research has high practical value. 


\section{Abbreviations}

LTS: Light translucent slit

BM: Black matrix

LBS: Light blocking slit.

\section{Data Availability}

All the data used to support the findings of this study are included within the article.

\section{Conflicts of Interest}

The authors declare no conflicts of interest.

\section{Acknowledgments}

This work was supported by the Sichuan Province Science and Technology Plan Project under Grant no. 2021YJ0094.

\section{References}

[1] Q. Kai-huai and J.-1. Luo, "Techniques for autostereoscopic display and its development," Journal of Image and Graphics, vol. 14, no. 10, pp. 1934-1941, 2009.

[2] Y. Jia-chen, H. Chun-ping, and L. Jian-jun, "Stereo image coding algorithm based on mesh," Journal of Optoelectronics-Laser, vol. 21, no. 1, pp. 136-139, 2010.

[3] T. Okosi, Three-Dimensional Imaging Techniques, Academic Press, Cambridge, MA, USA, 1976.

[4] N. A. Dodgson, "Autostereoscopic 3D displays," Computer, vol. 38, no. 8, pp. 31-36, 2005.

[5] Z. Ji-gui, X. Ting, and B. Wu, "Study on the coding design and point matching of LCD coded raster," Journal of Optoelectronics-Laser, vol. 16, no. 2, pp. 164-166, 2005.

[6] W. Mphepo, Y.-P. Huang, and H.-P. D. Shieh, "Enhancing the brightness of parallax barrier based $3 \mathrm{D}$ flat panel mobile displays without compromising power consumption," Journal of Display Technology, vol. 6, no. 2, pp. 60-64, 2010.

[7] J. Geng, "Three-dimensional display technologies," Advances in Optics and Photonics, vol. 5, no. 4, pp. 456-535, 2013.

[8] H. Yamamoto, H. Nishimura, T. Abe, and Y. Hayasaki, "Large stereoscopic LED display by use of parallax barrier of aperture grille type (Invited Paper)," Chinese Optics Letters, vol. 12, no. 6, pp. 060006-060010, 2014.

[9] S. Ping, A. Peng-li, and G.-f. Jin, "Demonstration of an autostereoscopic three-dimensional light-emitting diode display using diffractive optical elements sheet," Journal of Display Technology, vol. 11, no. 11, pp. 953-958, 2015.

[10] S. Ping, A. Peng-li, and C. Liang-cai, "Characteristics of the autostereoscopic three-dimensional LED display based on diffractive optical elements sheet," SPIEL, vol. 10022, p. 100221Y, 2016.

[11] A. N. Shu, S. U. Ping, M. A. Jian-she, and H. E. Ze-hao, "Analysis of stereo depth for 3D LED autostereoscopic displays based on the physical limitation," SPIEL, vol. 10378, p. 103780V, 2017.

[12] Y.-z. Huang, M. Rafiq Swash, and A. Sadka, "Innovative 3D pixel mapping method for LED holoscopic 3D display," SPIN, pp. 330-333, 2017.

[13] Z. Ren-liang, Z. Wu-xiang, and Q.-h. Wang, "Research on stereo viewing zone in autostereoscopic display based on parallax barrier," Acta Photonica Sinica, vol. 37, no. 5, pp. 960-963, 2008.

[14] H. Yamamoto, T. Kimura, S. Matsumoto, and S. Suyama, "Viewing-zone control of light-emitting diode panel for stereoscopic display and multiple viewing distances," Journal of Display Technology, vol. 6, no. 9, pp. 359-366, 2010.

[15] S. Xian-yu and L. Ji-tao, "Method for 3-D object recognition based on moire fringe," Journal of Optoelectronics laser, vol. 3, 2005.

[16] H.-x. Wang, S. Xian-yu, and L. Jing, "Method for 3D object recognition based on moiré fringe," Journal of Optoelectronics.Laser, vol. 16, no. 3, pp. 349-353, 2005.

[17] K. Ling-sheng, G. Jin, and T.-c. Wang, "Analysis of Moiré minimization in autostereoscopic parallax displays," Optics Express, vol. 21, no. 22, pp. 26068-26079, 2013.

[18] Y.-q. Wang, "Auto-stereoscopic display based on LCD," Chinese Journal of Liquid Crystals and Displays, vol. 18, no. 2, pp. 116-120, 2003.

[19] W. Pei-shun, Y. Jian-min, and G. Tai-liang, "Design of vertically staggered barrier for autostereoscopic display," Journal of Optoelectronics.Laser, vol. 22, no. 6, pp. 827-830, 2011.

[20] J. M. Yao, Q. Xin, and T. L. Guo, "Design of zigzag staggered barrier for autostereoscopic display," Acta Photonica Sinica, vol. 41, no. 10, pp. 1176-1179, 2012.

[21] X. Guo, H. Chun-ping, and S. Li-li, "Theoretical analysis of title angles based on autostereoscopic display with slanted barrier," Laser Technology, vol. 40, no. 3, pp. 388-391, 2016.

[22] Z. Xiang-Yao, Z. Xiong-Tu, and G. Tai-liang, "Crosstalk reduction in large-scale autostereoscopic 3D-LED display based on black-stripe occupation ratio[J]," Optics Communications, vol. 389, no. 15, pp. 159-164, 2017.

[23] L. S. Kong, C. Y. Liu, Y. Zhang, and G. Jin, "Progress on removing Moiré patterns in parallax autostereoscopic displays," Chinese Journal of Liquid Crystals and Displays, vol. 29, no. 3, pp. 441-449, 2014.

[24] Q.-h. Wang, 3D Display Technology and Devices, Science Press, Beijing, China, 2011. 